\title{
GCU
}

Glasgow Caledonian

University

University for the Common Good

\section{Indoor localization based on multiple LEDs position estimation}

Popoola, Olaoluwa Rotimi; Ogunkoya, Funmilayo Bimpe; Popoola, Wasiu O.; RamirezIniguez, Roberto; Sinanovic, Sinan

Published in:

IEEE 17th International Workshop on Signal Processing Advances in Wireless Communications (SPAWC)

DOI:

10.1109/SPAWC.2016.7536790

Publication date:

2016

Document Version

Author accepted manuscript

Link to publication in ResearchOnline

Citation for published version (Harvard):

Popoola, OR, Ogunkoya, FB, Popoola, WO, Ramirez-Iniguez, R \& Sinanovic, S 2016, Indoor localization based on multiple LEDs position estimation. in IEEE 17th International Workshop on Signal Processing Advances in Wireless Communications (SPAWC). IEEE, Edinburgh, UK. https://doi.org/10.1109/SPAWC.2016.7536790

\section{General rights}

Copyright and moral rights for the publications made accessible in the public portal are retained by the authors and/or other copyright owners and it is a condition of accessing publications that users recognise and abide by the legal requirements associated with these rights.

Take down policy

If you believe that this document breaches copyright please view our takedown policy at https://edshare.gcu.ac.uk/id/eprint/5179 for details of how to contact us. 


\title{
Indoor Localization based on Multiple LEDs Position Estimation
}

\author{
O. R. Popoola*, F. B. Ogunkoya*, W. O. Popoola ${ }^{\dagger}$, R. Ramirez-Iniguez* and S. Sinanović* \\ ${ }^{*}$ School of Engineering and Built Environment, Glasgow Caledonian University, Glasgow, G4 0BA, UK \\ Email: \{olaoluwa.popoola, funmilayo.ogunkoya, roberto.ramireziniguez, sinan.sinanovic\}@gcu.ac.uk \\ ${ }^{\dagger}$ Institute for Digital Communications, University of Edinburgh, Edinburgh, EH9 3JL, UK \\ Email:W.Popoola@ed.ac.uk
}

\begin{abstract}
This paper describes the simulation results and hardware implementation of an inexpensive, low-complexity LED based indoor positioning system. Localization by multiple LEDs estimation model (MLEM) approximates position of a mobile receiver by the acquisition of positional information from LED transmitters. Multiple LED orientation can either be with or without overlap. Receivers in a no-overlap LED orientation experience only single access while multiple access receivers are designed for orientations with overlaps. Single and multiple access systems were developed and implemented by the use of low cost ATMEG 328 microcontroller. Since multiple LEDs transmit data at the same wavelength and are asynchronous, overlap in multiple access system causes interference. The possibility of this interference is reduced by packet based pulse duration multiplexing (PDM) and a low duty cycle transmission protocol. By the use of MLEM, root mean square error in position estimation is reduced to about 1 percent of the length an indoor location. Experimental results show that overlap increases positional accuracy over a wider coverage region and that the multiple access system allows for a more reliable positioning.
\end{abstract}

Keywords- Indoor localization; optical wireless communications; Multiple LEDs; Microcontroller-based positioning; Overlap

\section{INTRODUCTION}

Indoor positioning and tracking has been the subject of exploration in a number of recent publications [1]. This is due to its applicability in various areas of life which include security, medicine, emergency rescue, object localization and tracking, system delivery, indoor mapping and trajectory generation [2]. Various methods for indoor positioning that have been investigated include object localization by the use of global positioning systems (GPS), radio frequency identification (RFID), ultrasound, infrared and wireless local area networks (WLAN). GPS has been used extensively for accurate positioning outdoors but, due to severe signal attenuation by walls and other obstacles, GPS signals greatly suffer degradation in indoor environments and hence are not effective for use in indoor tracking systems [1]. Indoor localization by ultrasound, WLAN, and RFID require additional infrastucture to operate [3].
Recently, with the current rise in Light Emitting Diodes (LEDs) as an effective method for lighting [4], LED based indoor positioning systems have been studied as a viable alternative to other aforementioned methods. This is because LED positioning has higher accuracy, no electromagnetic interference, higher security and higher capacity for data transmission [5], [6].

LED based positioning involves the use of known coordinates of LED transmitters to derive the unknown coordinates of an optical receiver which is usually a photo-detector (PD). The PD is used to acquire and translate information sent from LEDs. The acquired data is then analysed by appropriate algorithms to define the position of the receiver. The process is illustrated in Fig. 1. The sent signals from the LEDs however, suffer from a number of distortive effects which include multipath propagation, noise, shadowing and interference from other LED sources [7]. These distortive effects have posed challenges to developing a system for indoor positioning. Thus, a number of researchers have recently developed various algorithms to mitigate these effects.

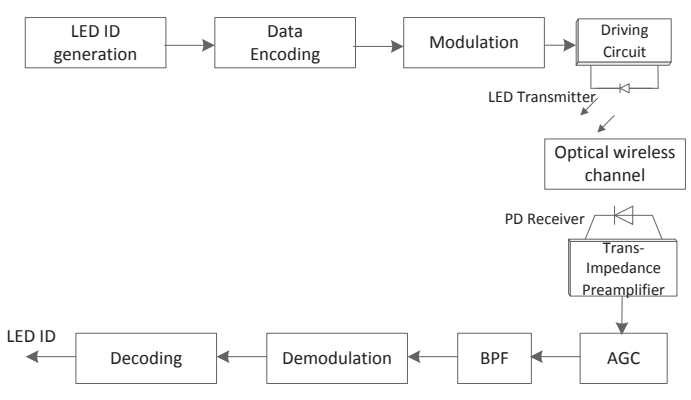

Fig. 1. Optical link design for a single transmitter and single receiver link. AGC: Automatic Gain Control; BPF:Band Pass Filter

Most of the papers published so far have explored methods that are based on received signal strength (RSS), angle of arrival (AOA) [1], time based multiplexing, frequency based multiplexing [8], receiver orientation modification [9], code division multiple access [10], and multiple optical receivers 
[11]. In addition, current trends have investigated LED based indoor positioning by RF carrier allocation [12], the use of smartphones [13], and location fingerprinting [14]. Heading estimation by the use of smartphone uses accelerometers, magnetometers and gyroscopes to track the movement of a receiver indoor by approximating the motion profile of the receiver module. This method is still being improved for real life scenarios while tracking by location fingerprinting requires complex algorithms. RSS and AOA methods have attempted to imitate successes in GPS systems by the use of trilateration and/or triangularization. These methods have been simulated to perform well. However, it is assumed that optical power from one source can be sufficiently separated from that of another source and that the received power, proportional to luminous intensity shown in Fig. 2a, is unique to a specific point. Nevertheless, the received power from an LED is not unique to a particular point but is rather the same for an infinite number of points on any contour as shown in Fig. 2b. To simplify designs with these algorithms, specific heights or angles are assumed in their simulation but a practical mobile receiver is not constrained by a particular height or angle [12], [9]. These assumptions make it difficult to implement the algorithms in practice and when implemented, the algorithms are complex and either expensive to implement or computationally intensive. Hence there is need to design a simple LED based positioning system which operates in real life scenario.

In this paper, we take advantage of interfering light beams to improve the accuracy of a LED based positioning system. We investigate possible position estimation error and examine various access systems perceivable by the receiver module. By using realistic assumptions, we develop a prototype from inexpensive off the shelf components.

The rest of the paper is organized as follows: description of the proposed positioning model is presented in Section II. In Section II-A and II-B, various configurations for the system design are discussed. Experimental findings and discussion on the performance of the proposed system are given in Section III. Finally, conclusions are presented in Section IV.

\section{Multiple LED Estimation Model (MLEM)}

MLEM uses both the content and pattern of reception of data carrying packets from single or multiple LEDs to directly infer the position of the receiver. Photons from an LED source travel with an irradiation angle which determines the spread of the beam from the source. This spread is used to arrange the LEDs so that irradiation from the source allows possibility of overlap with nearest neighbours. To infer position, a receiver in a position $x(t)$ at a time $t$ identifies specifically which optical transmitter(s) are in the region of reception of the receiver module. If at the time $t_{1}$, the receiver is in a region of no overlap and receives data from the transmitter at $\left(x_{t_{1}}, y_{t_{1}}\right)$, the receiver is said to be at the point $\left(x_{t_{1}}, y_{t_{1}}\right)$. Based on this, by the use of multiple LEDs, the receiver identifies multiple locations. The proposition of this model is that the smaller the distance between LED sources, the lower will be the positioning error. We investigate the viability of this model under various configurations as explained in Section II-A and II-B. By this method, the only condition for positioning is whether or not the signal from the LED, is received.

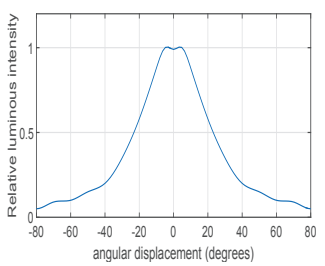

(a) Intensity levels at various angular displacement

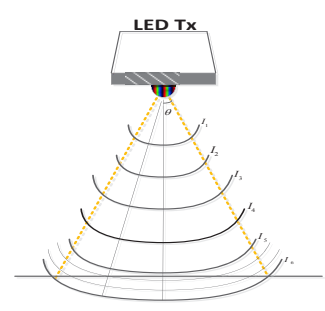

(b) contours illustrates points of equal luminous intensity
Fig. 2. Optical transmitter (LED) propagation characteristics. Source: [15], [16]

If this signal is not received, then the user is definitely in a non-coverage region. The non-coverage region is a region not covered by the modulated signal used for positioning. For a long, narrow area such as a hallway or a passage, Fig. 3 shows three possible scenarios between the light beams. For this work, a beam is the light coverage region where the LED light is above a detectable level.

Real life observations and practical light designs ensure that there are no non-coverage regions in indoor lighting. Consequently, in indoor positioning by MLEM, the mobile receiver is either at a location where there is no overlap between data packets or in a location where there is overlap as illustrated in Fig. 3a and $3 \mathrm{~b}$ respectively. These two conditions are considered as single access (no overlap) systems and multiple access (overlapping) systems. MLEM receivers are designed and investigated to determine positioning accuracy of the model as subsequently presented.
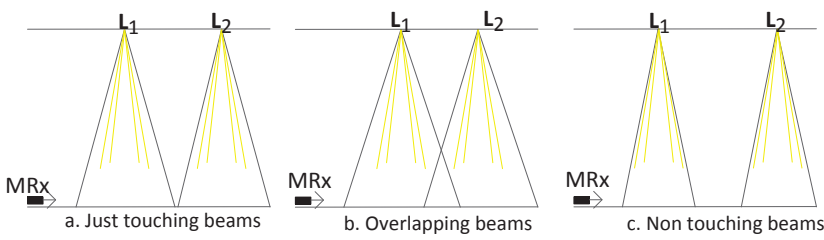

Fig. 3. Possible interaction between light beams from similar LED sources. $M R_{x}$ : Mobile receiver, $L_{1}$ : First LED source, $L_{2}$ : Second LED source.

\section{A. Single Access System}

The single access system is setup to investigate the feasibility of a low-cost LED-based indoor positioning system. The optical channel considered in this work is the line-of-sight (LOS) links. Single-access in this context means receiving position information from either of two LEDs with nonoverlapping beam of light. Each LED transmits a unique 8-bit code which contains its coordinates. The LEDs is designed to transmit this position information modulated using Pulse Amplitude Modulation (PAM) format to keep the algorithm 
simple. Thus, the radiated optical signal is linearly proportional to the amplitude of the electrical signal. To estimate the unknown position of the receiver, the received optical signal is converted back to proportionate electrical signal by the photodetector. The logical 0 s and 1 s of the 8-bits unique code are determined based on the received signal amplitude at each bit period. Thereafter, the receiver compares the estimate of the unique code with the known codes in its database in order to decipher the current position in real time.

For a channel with impulse response $h(t)$, the received optical data is converted to a proportionate current by the photodiode. This current signal $y(t)$ based on the modulated optical signal $x(t)$ is given in [5] by

$$
y(t)=R x(t) \otimes h(t)+N(t)
$$

where $R$ is the responsivity of the photodetector, $N(t)$ is the noise function, and $\otimes$ denotes convolution.

\section{B. Multiple Access System}

Data transmission via PAM suffices for the single access system. However, in the overlap region, since all transmitters are designed to transmit at the same wavelength, at the same time and in the same space, interference may occur. The interference in this region could either be from other LEDs, from ambient light or stray radiation from a similar device. Positional information to the LED is encoded using pulse duration multiplexing (PDM) to reduce possibility of interference by ambient light and stray devices. Encoding by PDM represents $1 \mathrm{~s}$ and $0 \mathrm{~s}$ by altering the duration of a pulse. This encoded data is modulated to a higher frequency using on-off keying (OOK) modulation. The high frequency (modulated) signal is transmitted through individual LED transmitters. If the signal transmitted from LED $L_{1}$ is $x_{1}(t)$ and $x_{2}(t)$ from LED $L_{2}$, the received current signal $y(t)$ is given by

$$
y(t)=R\left(x_{1}(t) \otimes h_{1}(t)+x_{2}(t) \otimes h_{2}(t)\right)+N(t)
$$

where $h_{1}(t)$ and $h_{2}(t)$ are the channel response from $L_{1}$ and $L_{2}$ respectively. Asynchronous data transmission is used to keep MLEM simple and inexpensive. Hence there is possibility of interference between bits of different transmitters. To reduce this interference, the modulated data are transmitted in short packets. This packet embeds the LED ID in a packet consisting of start bits, encoded data bits and a stop bit as shown in Fig. 4.

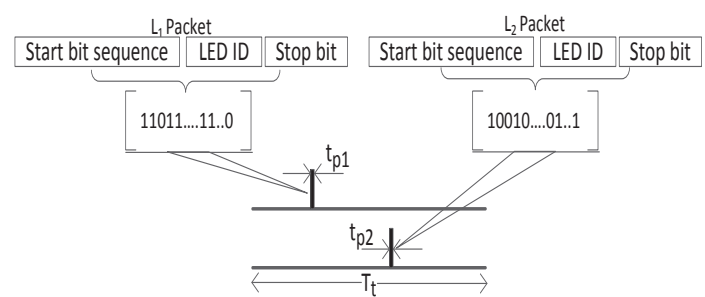

Fig. 4. Illustration of data packets formation and low duty cycle to reduce probability of collision.
The code packet for each LED transmitter is sent in a short pulse. The receiver recognises the start bit sequence in the packet and starts decoding. For a scenario with possibility of overlap between two LEDs, illustrated in Fig. 4, the duration of the pulse is given by $t_{p 1}$ for the first LED transmitter $L_{1}$ and $t_{p 2}$ for the second LED transmitter $L_{2}$. The total period for transmitting a sequence of code is given by $T_{t}$. The packet is transmitted with a low duty cycle $\left(t_{p 1}\right.$ and $t_{p 2}$ is far less than $T_{t}$ ) to reduce collision with packets from other LED transmitters. For $L_{1}$, the packet duty cycle $D C_{1}$ is given by the fraction $D C_{1}=\frac{t_{p 1}}{T_{t}}$.

For a duty cycle $D C_{1} \stackrel{t}{=} D C_{2}=\frac{1}{N} \quad \forall N \geqslant 2$, the probability of collision $P_{c}$ of packets from $L_{1}$ by those from $L_{2}$ is the probability of packets from the $L_{2}$ being in the region of the packets from $L_{1}$ as the same time. This is mathematically illustrated as:

$$
P_{c}=N \int_{0}^{\frac{1}{N}} f_{p_{1}}(x) d x \int_{\frac{-1}{N}}^{\frac{1}{N}} f_{p_{2}}(x) d x,
$$

where $f_{p_{2}}(x)$ and $f_{p_{2}}(x)$ represents the rectangular pulses from $L_{1}$ and $L_{2}$ respectively. For normalized pulse amplitudes, (3) reduces to

$$
P_{c}=\frac{2}{N} \text {. }
$$

In our design packet sizes and period were kept constant and the duty cycle ratio was kept at about 0.1 . The probability of collision is therefore given by $P_{c}=0.2$. Consequently, the probability of collision of packets from two LED sources is reduced to 2 out of every 10 packets transmitted.

\section{RESUlTS AND Discussions}

In this section, we present results obtained via simulation using MATLAB ${ }^{\circledR}$ communications toolbox and hardware implementation of the LED-based indoor localization system. Simulation is used to compare the positioning error in single access and multiple access systems. To quantify the results, we used the normalized Root Mean Squared Error (RMSE) metrics given by:

$$
R=\frac{\sqrt{\frac{1}{n} \sum_{i=1}^{n}\left(x_{t}^{(i)}-x_{p}\right)^{2}}}{L},
$$

where $L$ is the total distance covered by light from LEDs, $n$ is the number of iterative points, $x_{t}^{(i)}$ is the true location of the receiver at any iterative point $i$ and $x_{p}$ is the location of the transmitter which represents the estimated position of the receiver. The optimal design conditions obtained from the simulations are implemented in hardware and performance of the system with overlapping and non-overlapping beams evaluated.

\section{A. Simulation Results and discussions}

The systems under consideration here are shown in Fig. 3a (Case 1) and 3b (Case 2). For Case 1, a mobile receiver is placed at a point in the coverage region and moved in small discrete intervals. Positioning error is calculated as the deviation of the ascribed receiver location (the LED 
coordinates received) from the true location of the receiver. The RMSE is obtained using (5). The process is repeated for increasing number of LEDs and the results are shown in Fig. 5. For Case 2, the distance between the LEDs determined the level of overlap the at the receiver. The effect of the level of overlap on the positioning error is carried out by varying the distance between LEDs, thereby causing overlaps, and measuring the error. It is observed that minimum positioning error occurred when the distance of overlap is equal to the distance of no overlap. Using this condition, positioning error is calculated by a similar process as described in Case 1 and results presented in Fig. 5. These two systems are compared and the positioning error for both is seen to reduce as the number of LEDs increases. It is observed that by the use of MLEM, with 10 LEDs, the positioning error can be reduced to $2.63 \%$ of the total length of the passage for just touching beams and $1.05 \%$ of the length of the passage for overlapping beams. Generally, by the effective use of overlap in Case 2, the error in positioning is reduced by about $50 \%$ and better accuracy figures could be obtained by increasing the number of LEDs used.

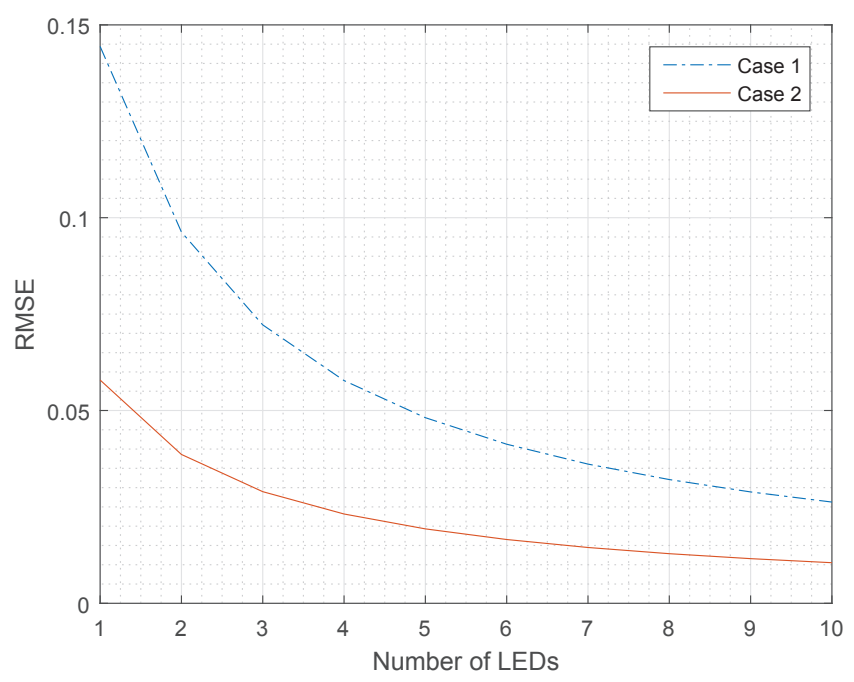

Fig. 5. Variation of RMSE vs Number of LEDs for Case 1 and Case 2. Case 1: Just touching beams (Fig. 3a) and, Case 2: Overlapping beams (Fig. 3b)

\section{B. Experimental Results and discussions}

For laboratory experiments single and multiple access systems are developed using ATMEG 328 microcontrollers and positioned as shown in Fig. 3. Height, which is the vertical distance between the plane of the LEDs and the plane of the mobile receiver, is kept at $30 \mathrm{~cm}$ due to workbench space constraint but the developed hardware has been tested to deliver data at a height of about $4 \mathrm{~m}$. Other hardware specifications for the experiments are given in Table I. Results from the experiment are quantified by measuring the Packet Delivery Ratio (PDR) given by:

$$
P D R=\frac{P k_{r}}{P k_{s}}
$$

where $P k_{r}$ is the number of packets received correctly and $P k_{s}$ is the number of packets sent. For a single access system, Fig. 6 shows the variation of PDR with angular displacement as the mobile receiver travels on a particular horizontal plane parallel to the plane of the LEDs as illustrated in Fig. 3. As expected, full packet delivery is obtained at the perpendicular from the LED to the horizontal plane and a region at angular displacement of $\pm 5^{\circ}$ to this perpendicular.

TABLE I

PARAMETERS FOR EXPERIMENTAL SETUP

\begin{tabular}{cl}
\hline \multicolumn{2}{c}{ Parameters } \\
\hline Light emitting diode (LED) & OSRAM IR LED (SFH 4550) \\
angle of half intensity $\varphi_{1 / 2}$ & $\pm 3^{\circ}$ \\
peak wavelength $\lambda_{p}$ & $860 \mathrm{~nm}$ \\
total radiant flux $\phi_{a}$ & $70 \mathrm{~mW}$ \\
rise and fall time $t_{r}, t_{f}$ & $12 \mathrm{~ns}$ \\
Photodetector (PD) & VISHAY (TSOP 38238) \\
Directivity $(\theta)$ & $45^{\circ}$ \\
Peak wavelength $\lambda_{p}$ & $950 \mathrm{~nm}$ \\
Minimum irradiance $E_{(\text {emin })}$ & $\left.0.12 \mathrm{~mW} / \mathrm{m}^{2}\right)$ \\
Microcontroller & ATMEG 328 \\
Clock frequency & $16 \mathrm{MHz}$ \\
\hline
\end{tabular}

As the receiver moves away from this region, the PDR reduces. Furthermore, decrease in the distance between the plane of the LED and the receiver shows full packet delivery at a wider angular displacement of $\pm 20^{\circ}$ from the perpendicular. Therefore the region of reception of packets is affected by the distance between the transmitter and receiver plane. The larger the distance, the narrower the region of reception and this narrow region implies greater positioning accuracy (Section II). Thus MLEM does not assume a fixed height but, provided the receiver is in the region of coverage, the positioning accuracy is improved as the vertical distance between the transmitter and the receiver increases.

To determine the effect of overlap on position error, the transmitters, receiver system was arranged based on the angle of coverage of the transmitters given in Table I and the condition for minimal error in overlap (Section III-A). We measure the variation of PDR with linear displacement on the receiver horizontal plane for both single and multiple access system and the results are presented in Fig. 7 and 8 respectively.

The hardware is designed to send packets every $200 \mathrm{~ms}$. Hence for successful packet delivery every seconds a minimum of 1 out of every 5 packets sent must be correctly decoded. Thus a PDR above 0.2 is required. In Fig. 7 there is a region of no coverage (PDR below 0.2) between the two LEDs hence the position of a receiver in this region is unknown. This situation is taken care of by the overlap design shown in Fig. 8 where the PDR is almost above average (0.5) in all the region of coverage. Packet reception occurs for $66.6 \%$ of displacement in the single access and for $86.6 \%$ in the multiple access system. It is observed that packet loss, given by low PDR, 


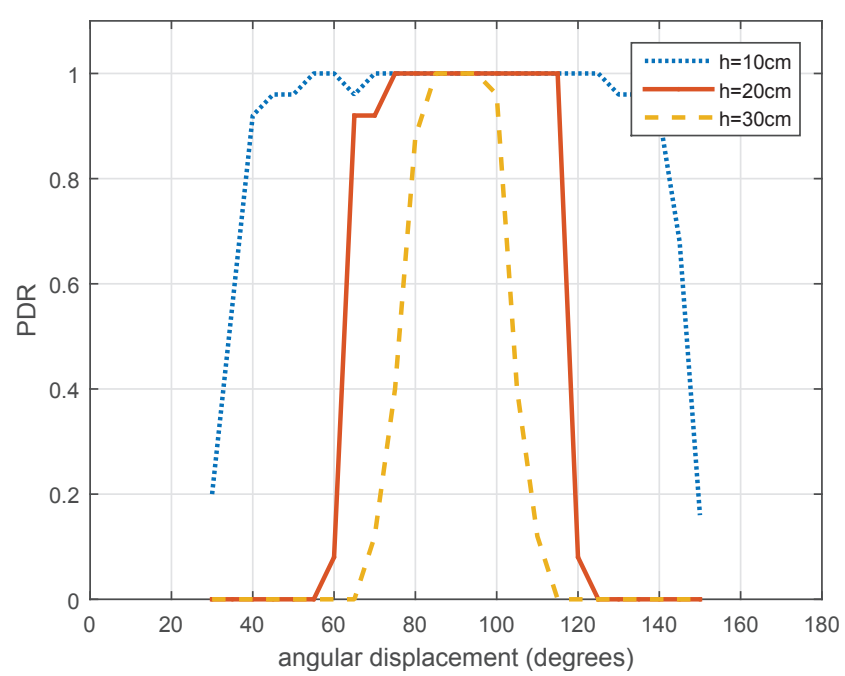

Fig. 6. Variation of PDR with angular displacement of receiver at various distances from a transmitter.

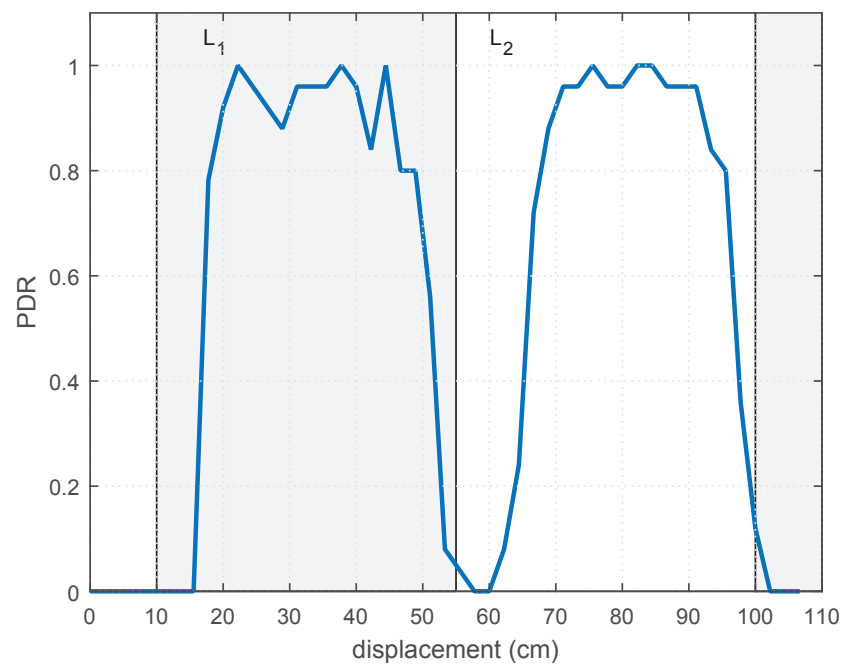

Fig. 7. Variation of PDR with displacement for single access system of two LEDs $L_{1}$ :coverage region for LED 1 and $L_{2}$ : coverage region for LED 2 .

happens more just at the boundaries near the overlaps (Fig. 8) where the receiver has to distinguish between being in a region of overlap or not. By (5), the RMSE value for the system with overlap is calculated to be 0.069 while the non overlapping system has an RMSE of 0.12 . These RMSE values are slightly more when compared to the simulation based RMSE values for two LEDs because ideal conditions are assumed in simulation.

In addition, it is also observed that the single access system identifies just two positions ( $L_{1}$ and $L_{2}$ ) for two LEDs, whereas the multiple access systems identifies an extra 'overlap' region. As the number of LEDs increases, the multiple access system identifies more overlapping regions and thereby improves the accuracy of positioning. Thus, MLEM takes advantage of overlap to improve the reliability of the system by offering a better resolution of positioning, better coverage and lower RMSE.

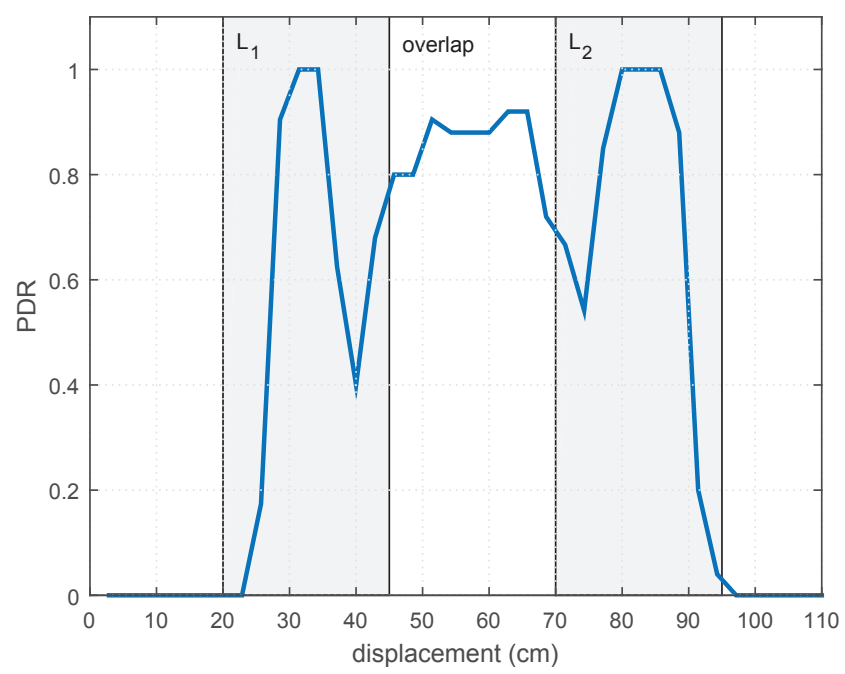

Fig. 8. Variation of PDR with displacement for multiple access system of two LEDs (overlap). $L_{1}$ :coverage region for LED 1 and $L_{2}$ : coverage region for LED 2. overlap indicates the overlap region between $L_{1}$ and $L_{2}$.

\section{CONCLUSION}

In this paper, a microcontroller-based LED indoor positioning system using MLEM is described. The system is designed to be compact and inexpensive by the use of simple positioning algorithm. The paper determines RMSE values for an indoor location using MLEM in both single access and multiple access LED transmitter configurations. By the use of pulse duration multiplexing to reduce interference, the paper demonstrates how optical signals can be transmitted in the same frequency, space and time. Therefore, overlap which should normally cause packet loss is used to improve the reliability of positioning and reduce positioning error thus making the system more accurate and applicable to real life situations. Data packets carrying transmitter coordinates are correctly decoded at the receiver irrespective of whether there is overlap or not.

In future works, the applicability of this positioning technique to more general scenarios would be investigated and a detailed research of multipath reflections on signal decoding will be considered.

\section{REFERENCES}

[1] S.-H. Yang, H.-S. Kim, Y.-H. Son, and S.-K. Han, "Three-dimensional visible light indoor localization using AOA and RSS with multiple optical receivers," Journal of Lightwave Technology, vol. 32, no. 14, pp. 2480-2485, 15 July 2014.

[2] D. Dardari, P. Closas, and P. M. Djuric, "Indoor tracking: Theory, methods, and technologies," IEEE Transactions on Vehicular Technology, vol. 64, no. 4, pp. 1263-1278, April 2015.

[3] A. Yazici, U. Yayan, and H. Yücel, "An ultrasonic based indoor positioning system," in International Symposium on Innovations in Intelligent Systems and Applications (INISTA), Istanbul, 15-18 June 2011, pp. 585589.

[4] S. Babadi, R. Ramirez-Iniguez, T. Boutaleb, and T. Mallick, "Performance analysis of a DTIRC-LED illumination structure," in Proceedings SPIE, Optics and Measurement Conference, vol. 9442, Liberec, Czech Republic, 7-10 October 2014. 
[5] X. Zhang, J. Duan, Y. Fu, and A. Shi, "Theoretical accuracy analysis of indoor visible light communication positioning system based on received signal strength indicator," Journal of Lightwave Technology, vol. 32, no. 21, pp. 3578-3584, 2014.

[6] S.-H. Yang, E.-M. Jeong, D.-R. Kim, H.-S. Kim, Y.-H. Son, and S.$\mathrm{K}$. Han, "Indoor three-dimensional location estimation based on LED visible light communication," Electronics Letters, vol. 49, no. 1, pp. 54-56, 2013.

[7] A. M. Street, P. N. Stavrinou, D. C. O'brien, and D. J. Edwards, "Indoor optical wireless systems-a review," Optical and Quantum Electronics, vol. 29, no. 3, pp. 349-378, 1997.

[8] U. Nadeem, N. U. Hassan, M. A. Pasha, and C. Yuen, "Indoor positioning system designs using visible LED lights: performance comparison of TDM and FDM protocols," Electronics Letters, vol. 51, no. 1, pp 72-74, 8 January 2014

[9] T. Huang, X. Gao, Y. Guo, S. Li, Q. Li, C. Li, H. Zhu, and Y. Wang, "Visible light indoor positioning fashioned with a single tilted optical receiver," in 14th International Conference on Optical Communications and Networks (ICOCN), Nanjing, 3-5 July 2015, pp. 1-4.

[10] S. De Lausnay, L. De Strycker, J.-P. Goemaere, N. Stevens, and B. Nauwelaers, "Optical CDMA codes for an indoor localization system using VLC," in 3rd International Workshop in Optical Wireless Communications (IWOW), Funchal, 17 September 2014, pp. 50-54.
[11] M. Yasir, S.-W. Ho, and B. Vellambi, "Indoor position tracking using multiple optical receivers," Journal of Lightwave Technology, vol. 34, no. 4, pp. 1166-1176, 15 February 2016.

[12] H.-S. Kim, D.-R. Kim, S.-H. Yang, Y.-H. Son, and S.-K. Han, "An indoor visible light communication positioning system using a RF carrier allocation technique," Journal of Lightwave Technology, vol. 31, no. 1, pp. 134-144, 1 January 2013.

[13] W. Kang, S. Nam, Y. Han, and S. Lee, "Improved heading estimation for smartphone-based indoor positioning systems," in IEEE 23rd International Symposium on Personal Indoor and Mobile Radio Communications (PIMRC), Sydney, NSW, 9-12 September 2012, pp. 2449-2453.

[14] K. Kaemarungsi and P. Krishnamurthy, "Modeling of indoor positioning systems based on location fingerprinting," in Twenty-third Annual Joint Conference of the IEEE Computer and Communications Societies (INFOCOM), vol. 2. IEEE, 7-11 March 2004, pp. 1012-1022.

[15] Vishay Semiconductors, "Datasheet: TLUR6400 Universal LED in 5 mm Tinted Diffused Package," Retrieved February 2, 2016 from http://www.vishay.com/docs/83055/tlur540.pdf, October 2012.

[16] J. M. Kahn and J. R. Barry, "Wireless infrared communications," Proceedings of the IEEE, vol. 85, no. 2, pp. 265-298, 1997. 